\title{
A numerical investigation of the effects of metal foam characteristics and heating/cooling conditions on the phase change kinetic of phase change materials embedded in metal foam
}

\author{
Mohamed Moussa EL IDI*, Mustapha KARKRI \\ Université Paris Est, CERTES. \\ 61 Av. du Général de Gaulle, 94010 Créteil Cedex, France. \\ * Corresponding author: moussa.elidi@gmail.com
}

\begin{abstract}
In the last decade, the use of Phase Change Materials (PCM) as passive thermal energy storage has been widely studied both numerically and experimentally. Despite their advantages, their thermal conductivity is very low, with a large change in volume during the melting and solidification process. One way to increase their poor thermal conductivity is to embed them into open cells metallic foams. In this paper, a numerical study is conducted on the effects of metal foam properties and heating/cooling conditions (cyclic heating and cooling) on the phase change kinetic of PCM embedded in metal foam. Two types of heating condition were studied: constant and sinusoidal heat flux. The mathematical model is based on the volume-averaging technique. The Brinkman-Forchheimer-extended Darcy equation and the local thermal non-equilibrium model that applies a two-energy equation are used. The phase change of PCM is modeled by an enthalpy-porosity method. The numerical results are validated by comparing with experimental data. It is observed from the numerical results that the foam morphology and materials have a significant influence on the melting and solidification of phase change materials. The results also show that the melting time is reduced with sinusoidal heating.
\end{abstract}

Keywords: Thermal energy storage, Numerical simulation, Phase change material, Metal foam.

$\begin{array}{llll}C_{P} & \text { Specific heat capacity, }\left(\mathrm{J} \cdot \mathrm{kg}^{-1} \cdot \mathrm{K}^{-1}\right) & C & \text { Inertial coefficient, }\left(W \cdot \mathrm{m}^{-2} \cdot \mathrm{K}^{-1}\right) \\ \rho & \text { Density, }\left(\mathrm{kg} / \mathrm{m}^{3}\right) & K & \text { Permeability, } \mathrm{m}^{2} \\ L_{f} & \text { Latent heat of fusion }(\mathrm{J} / \mathrm{kg}) & \text { subscripts } & \\ \mu & \text { Dynamic viscosity }\left(\mathrm{Pa}^{-1}\right) & s & \text { Metal foam } \\ t & \text { Time },(\mathrm{s}) & w & \text { Hot wall } \\ \beta & \text { Thermal expansion of } \mathrm{PCM},\left(K^{-1}\right) & f & \text { fluid } \\ T & \text { Temperature },(\mathrm{K}) & & \text { solid } \\ k & \text { Thermal conductivity, }(\mathrm{W} / \mathrm{m} . \mathrm{K}) & \mathrm{P} & \text { Phase change material }\end{array}$

\section{Introduction}

Phase change materials (PCMs) are widely used in many engineering applications [1] [2]. However, most of them have a low thermal conductivity, which reduces the rate of heat storage and extraction during their melting and solidification cycle. These factors limit the use of these materials [3]. Therefore, heat transfer enhancement techniques are required for a thermal energy storage system when using PCMs. The thermal conductivity of PCMs can be enhanced by the addition of some materials with high thermal conductivity, such as fins [4], porous graphite [5], nanoparticles [6], and high-porosity metal cell [6] [7]. Among these heat transfer enhancers, the high-porosity opencell metal foam is found to be especially effective in improving the performance of PCMs because of its high thermal conductivity, large surface area to volume ratio, highly interconnected structure and natural fluid mixing effect. Due to the wide applications of PCM/metal foam composite, the phase change characteristics in metal foam have attracted increasing research attention [8].

The kinetic of phase change is an important factor in the performance of PCM composites. Control of the phase change kinetic of PCM embedded in metal foam is possible via different parameters, such as the nature of PCM, the geometrical configuration, the metal foam properties, and the boundary conditions. Here, the emphasis is focused on 
the metal foam properties, and heating and cooling conditions in a continuous process (cyclic heating and cooling). Much research has conducted studies of the effects of metal foam thermal conductivity and microstructure on the effective thermal conductivity of PCM/metal foam composites. The effective thermal conductivity has a great impact on the phase change kinetic. If the effective conductivity is high, heat transfer will be intensified during melting and solidification and therefore phase change will be faster. However, it is more important to study the direct effect of the microstructure and thermal conductivity of the boundary condition foam on phase change kinetic, in other words, the evolution of the liquid fraction, which is the subject of this article. Recently, studies have been conducted on the effects of these parameters on kinetics and phase change kinetic, but not on the effect of heating and cooling conditions especially in a continuous process (cyclic heating and cooling). Xia et al. [9] also studied the effective thermal conductivity of paraffin/nickel foam composites and paraffin/copper foam composites with high metal foam porosity $(>95 \%)$. The study was carried out on the effect of porosity on the effective thermal conductivity of paraffin/metal foam composites. The results show that the effective thermal conductivity increases as the porosity decreases. Xiang Huang et al. [10] conducted an experimental study to investigate the thermal conductivity enhancement of Myristyl alcohol (MA) by impregnating metal foam. Two types of foam were investigated. It was found that the use of copper foam increases the effective thermal conductivity of MA/copper foam by 7.51 times and the use of nickel foam increases the effective thermal conductivity of PCM composite by 1.80 times compared to pure MA. Sriharsha S. et al. [11] carried out a numerical study on the effects of the pore size of aluminum foam on the effective thermal conductivity of paraffin/aluminum foam composite using finite element analysis. To study the effect of the pore size, an aluminum foam with 0.94 porosity and pore sizes varying from five to $100 \mu \mathrm{m}$ was considered. The results show that a smaller pore size results in higher thermal conductivity. On average, the thermal conductivity more than doubled from $5 \mathrm{~W} /(\mathrm{m} . \mathrm{K})$ to $12 \mathrm{~W} /(\mathrm{m} . \mathrm{K})$ when the pore size was reduced from $100 \mu m$ to $25 \mu m$. Y. Zhao et al. [12] carried out a numerical study on the effect of porosity and pore density on the melting rate and solidification rate. The phase change of PCM in metal foam was modeled using the phase field method. To study the effect of the structure of metal foam on melting and solidification, four metal foams with the same pore density of 20PPI and different porosities (between 0.80-0.95) were used. The same methodology was used to the study the effect of pore density, that is, four metal foams with the same porosity of 0.9 and different pore densities (5PPI, 10PPI, 20PPI and 40PPI). It was found that the foam's properties (porosity and pore density) play an essential role in the melting and solidification of PCM. The melt fraction of PCM embedded in a metal foam increases with decreasing porosity and increases with increasing pore density. S. Abishek et al. [13] studied the effect of the microstructure on the melting in a metal foam/paraffin composite. The authors modeled the phase change via the enthalpy-porosity method and used an additional volume fraction and field to account for the degree of melting. They used chtMultiRegionFoam in OpenFOAM, a conjugate heat transfer and fluid dynamics solver, to simulate the melting process in paraffin aluminum foam composites with a given porosity but a varying microstructure. The results show that the specific area, which is the ratio between surface and volume, plays an essential role in the melting of paraffin embedded in aluminum foam. This paperfocuses on the study of porosity, pore density and the thermal conductivity of metal foam to ascertain whether there is a direct relationship between the melting rate of PCM and metal foam thermal conductivity, and heating and cooling conditions on the phase change kinetic of PCM embedded in metal foam. The effects of porosity and pore density have already been studied by Y. Zhao et al. [12], who used a model that predicts the thermal behavior of PCM/metal foam composites, with less precision than our model (see Fig. 2). Then, we study the effect of heating and cooling conditions in a continuous process that has not been studied before in the literature. The study of effects of heating and cooling conditions on the kinetic of phase change will be very interesting, and will add to studies already included in the available literature and help to optimize the use of PCM/metal foam for a given application. One of the important applications of MCP composites is the cooling of certain electronic devices that generate a constant heat flux and passive solar energy storage which is affected by the variation in solar radiation. It is for these reasons that constant and sinusoidal heating and cooling have been chosen as the focus of this study. For constant heat flux, a step function ranging from $+1800 \mathrm{~W} / \mathrm{m}^{2}$ to $-1800 \mathrm{~W} / \mathrm{m}^{2}$ was considered. For variable heat flux, a sine function with a similar area as the step function was considered for one wall of the container to provide heating and cooling to the PCM/metal foam composite.

In this paper, a numerical study on metal foam properties is presented: porosity, pore density and thermal conductivity, and the cyclic heating and cooling effects on the kinetic of phase change. The mathematical model is based on the volume-averaging technique. The Brinkman-Forchheimer-extended Darcy equation and the local thermal non-equilibrium model applying a two-energy equation are used. The phase change of PCM is modeled by the enthalpy-porosity method. The numerical results are validated by comparing with experimental data. The effects of porosity, pore density and metal foam thermal conductivity are studied. The effects of heating/cooling conditions are also investigated.

\section{Modeling method}

2.1. Physical problem

The physical problem to be tackled is illustrated in Fig. 1. A square cavity with dimension $4 \mathrm{cmx} 4 \mathrm{~cm}$ is filled with paraffin wax RT27 embedded in a metal foam. The left wall cavity is set to a fixed temperature $T_{w}$, which is higher 
than the melting temperature $T_{m}$ in the melting process or lower than the melting temperature $T_{m}$ in the solidification process. The rest of the wall cavity is insulted. Initially, the metal foam and PCMs in the cavity are uniformly under the same melting temperature. The proprieties of PCM used for this study are given in Table 1 [14].

\begin{tabular}{|c|c|c|}
\hline Properties & Paraffin RT27 & Paraffin RT58 \\
\hline Density $\left(k g . m^{-3}\right)$ & 870 & 850 \\
\hline Heat capacity-solid $\left(J . K^{-1} \cdot \mathrm{kg}^{-1}\right)$ & 2400 & 2100 \\
\hline Latent heat $\left(K J . \mathrm{kg}^{-1}\right)$ & 179 & 181 \\
\hline Melting temperature $(K)$ & 300.15 & 331.15 \\
\hline Dynamic viscosity $\left(K g \cdot \mathrm{m}^{-1} \cdot \mathrm{s}^{-1}\right)$ & $3,42.10^{-3}$ & 0.0269 \\
\hline Heat conductivity -solid $\left(W \cdot K^{-1} \cdot m^{-1}\right)$ & 0.24 & 0.2 \\
\hline Density - liquid $\left(\mathrm{kg}^{\mathrm{m}} \mathrm{m}^{-3}\right)$ & 760 & 775 \\
\hline Heat capacity -liquid $\left(J . K^{-1} . \mathrm{kg}^{-1}\right)$ & 1800 & 2100 \\
\hline Heat conductivity - liquid $\left(W \cdot K^{-1} \cdot m^{-1}\right)$ & 0.15 & 0.2 \\
\hline$\beta\left(K^{-1}\right)$ & $0.5 \times 10^{-3}$ & $1.1 \times 10^{-4}$ \\
\hline
\end{tabular}

Table 1: Thermophysical properties of paraffin RT27 and RT58.

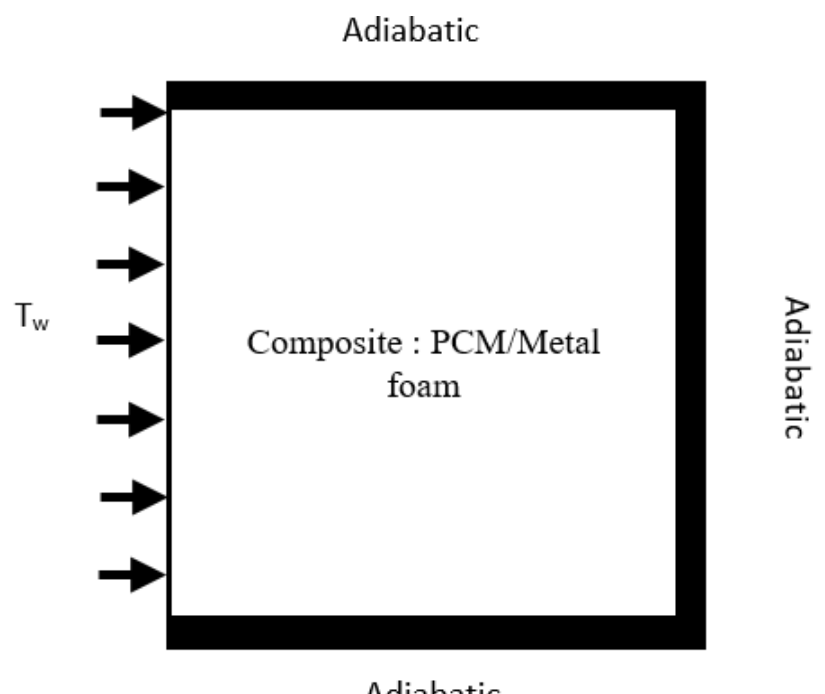

Fig. 1. Physical model

\subsection{Assumption}

- The liquid is considered as incompressible, and the flow is laminar.

- The natural convection caused by buoyancy is subject to the Boussinesq approximation.

- The thermal radiation is neglected in the metal foam during melting.

- The metal foam is considered as isotopic and homogeneous.

2.3. Mathematical model

In the model developed in this paper, a volume-averaging technique is employed [15]. Based on the above assumptions, the mathematical model for the present problem can be expressed by the following equations:

Continuity equation:

$$
\frac{\partial u}{\partial x}+\frac{\partial v}{\partial y}=0
$$

Momentum equations:

$$
\begin{gathered}
\frac{\rho_{P}}{\varepsilon} \frac{\partial u}{\partial t}+\frac{\rho_{P}}{\varepsilon^{2}}(\vec{V} \vec{\nabla}) \cdot u=-\frac{\partial p}{\partial x}+\frac{\mu}{\varepsilon} \nabla^{2} u-\left(\frac{\mu}{K}+\frac{\rho_{P} C}{\sqrt{k}}|u|\right) u+S_{u} \\
\frac{\rho_{P}}{\varepsilon} \frac{\partial v}{\partial t}+\frac{\rho_{P}}{\varepsilon^{2}}(\vec{V} \vec{\nabla}) \cdot v=-\frac{\partial p}{\partial y}+\frac{\mu}{\varepsilon} \nabla^{2} v-\left(\frac{\mu}{K}+\frac{\rho_{P} C}{\sqrt{k}}|v|\right) v+S_{v}+\rho_{P} g \beta\left(T-T_{r e f}\right)
\end{gathered}
$$

Where $S_{u}=A \frac{(1-b)^{2}}{B^{3}+b} u$, and $S_{v}=A \frac{(1-b)^{2}}{B^{3}+b} v$ denote the source term to damping the velocity in solidified phase ( $A$ and $B$ are two constants). In this study, we take $A=10^{5}$ and $B=10^{-3}$. They are deducted from the Carmen- 
Kozney relation [16] [17]. For the solidified phase, $b=0$ leaded to this item to be infinity to damp the velocity in the momentum equation. For fluid phase, $b=1$, leaded to the item to be zero.

$b(T)$ can be given as:

$$
b(T)=\left\{\begin{array}{lc}
0 & T<\left(T_{m}-\Delta T / 2\right) \\
\frac{T-T_{m}+\Delta T / 2}{\Delta T} & \left(T_{m}-\frac{\Delta T}{2}\right) \leq T<\left(T_{m}+\frac{\Delta T}{2}\right) \\
1 & T>\left(T_{m}+\Delta T / 2\right)
\end{array}\right.
$$

The dynamic viscosity is modified by using $S(T)=A \frac{(1-b)^{2}}{B^{3}+b}$ to have an extremely high value when the PCM is at a temperature below its melting temperature.

$$
\mu_{P}(T)=\rho_{P l}(1+S(T))
$$

There is a large difference in thermophysical properties between metal foam and saturating PCM [18]. There are two non-equilibrium equations:

Energy equation for PCM:

$$
\varepsilon \rho_{P} C_{P} \frac{\partial T_{f}}{\partial \tau}+\rho_{P} C_{P}\left(u \frac{\partial T_{P}}{\partial x}+v \frac{\partial T_{P}}{\partial x}\right)=k_{p} \nabla^{2} T_{P}+h_{P S} a_{P S}\left(T_{S}-T_{P}\right)
$$

The density variation of PCM can be given by:

$$
\rho_{P}(T)=\rho_{P s}+\left(\rho_{P l}-\rho_{P s}\right) \cdot b(T)
$$

The phase change temperature interval is taken as $3^{\circ} \mathrm{C}$ [14]. The specific heat of PCM can be written as [19] [20]:

$$
C_{P P}=C_{P s}+\left(C_{P l}-C_{P s}\right) \cdot b(T)+L_{f} D(T)
$$

Where ${ }_{L_{f}}$ is the latent heat of fusion and $D$ is a smoothed Delta Dirac function which is zero everywhere except in the interval [19]. Its integral is equal to 1 . The use of this function ensures that the latent heat is conserved during the melting process:

$$
D(T)=e^{\left(\frac{\frac{-\left(T-T_{m}\right)^{2}}{(\Delta T / 4)^{2}}}{\sqrt{\pi(\Delta T / 4)^{2}}}\right)}
$$

The thermal conductivity of PCM can be given as:

$$
k_{P}(T)=k_{P s}+\left(k_{P l}-k_{P s}\right) \cdot b(T)
$$

Energy equation for metal foam:

$$
(1-\varepsilon) \rho_{S} c_{P S} \frac{\partial T_{S}}{\partial \tau}=k_{S} \nabla^{2} T_{S}-h_{P S} a_{P S}\left(T_{S}-T_{P}\right)
$$

Where $k_{P_{e}}, k_{S_{e}}, h_{P S}$ are the effective thermal conductivity of paraffin, the effective thermal conductivity of metal foam and the interstitial heat transfer coefficient between the porous metallic surface and saturating PCM. Zukauskas' [21] semi-empirical model was used to estimate $h_{P S}$. Re is Reynolds number.

$$
h_{P S}=\left\{\begin{array}{cc}
0.76 \operatorname{Re}^{0.4} \operatorname{Pr}^{0.37} \frac{k_{P}}{d} & 0<\operatorname{Re} \leq 40 \\
0.52 \operatorname{Re}^{0.5} \operatorname{Pr}^{0.37} \frac{k_{P}}{d} & 40<\operatorname{Re} \leq 1000 \\
0.26 \operatorname{Re}^{0.6} \operatorname{Pr}^{0.37} \frac{k_{P}}{d} & 1000<\operatorname{Re} \leq 20000
\end{array}\right.
$$

Many models have been developed to describe the effective thermal conductivity. The tetrakaidecahedron cell model, proposed by Boomsma and Poulikakos [22], was adopted to describe the thermal conductivity in this study as shown in the following equations:

$$
R_{A}=\frac{4 d}{\left(2 e^{2}+\pi d(1-e)\right) k_{s}+\left(4-2 e^{2}-\pi d(1-e)\right) k_{f}}
$$




$$
\begin{gathered}
R_{B}=\frac{(e-2 d)^{2}}{(e-2 d) e^{2} k_{s}+\left(2 e-4 d-(e-2 d) e^{2}\right) k_{f}} \\
R_{C}=\frac{(\sqrt{2}-2 e)^{2}}{2 \pi d^{2}(1-2 e \sqrt{2}) k_{s}+2\left(\sqrt{2}-2 e-\pi d^{2}(1-e \sqrt{2})\right) k_{f}} \\
R_{D}=\frac{2 e}{e^{2} k_{s}+\left(4-e^{2}\right) k_{f}} \\
k_{e f f}=\frac{\sqrt{2}}{2\left(R_{A}+R_{B}+R_{C}+R_{D}\right)}
\end{gathered}
$$

Where $d=\sqrt{\frac{\sqrt{2}\left(2-(5 / 8) e^{3} \sqrt{2}-2 \varepsilon\right)}{\pi(3-4 e \sqrt{2}-e)}}$ and $\mathrm{e}=0.339$.

The effective thermal conductivity of PCM and metal foam can be defined as:

$$
\begin{aligned}
& k_{P e}=k_{\text {eff }} \mid k_{s}=0 \\
& k_{S e}=k_{\text {eff }} \mid k_{f}=0
\end{aligned}
$$

\section{Thermophysical and structural proprieties of metal foam}

The thermophysical proprieties of metal foam used in this study are presented in Table 2. Many kinds of research have been carried out to develop an analytical model for predicting the permeability and inertial coefficient of metal foam [23-29]. In this work, Calmidi's [25] model was used to calculate the permeability and inertial coefficient.

Permeability:

Inertial coefficient:

$$
K=0.00073 d_{p}^{2}(1-\varepsilon)^{-0.224}\left(\frac{d_{f}}{d_{p}}\right)^{-1.11}
$$

$$
C=0.00212 d_{p}^{2}(1-\varepsilon)^{-0.132}\left(\frac{d_{f}}{d_{p}}\right)^{-1.63}
$$

Where $d_{f}$ denotes the equivalent diameter of metal foam fibers and $d_{p}$ pores diameter [24]:

$$
\frac{d_{f}}{d_{p}}=1.18 \sqrt{\frac{1-\varepsilon}{\pi}}\left(\frac{1}{1-e^{-(1-\varepsilon) / 0.04}}\right)
$$

\begin{tabular}{|c|c|c|c|}
\hline Properties & Aluminum & Copper & Nickel \\
\hline Thermal conductivity $\left(W \cdot m^{-1} \cdot K^{-1}\right)$ & 220 & 401 & 70 \\
\hline Specific heat capacity $\left(J \cdot \mathrm{kg}^{-1} \cdot \mathrm{K}^{-1}\right)$ & 900 & 385 & 456 \\
\hline$\left(\right.$ kg. $\left.\mathrm{m}^{-3}\right)$ & 2700 & 8960 & 8890 \\
\hline
\end{tabular}

$d_{p}$ can be calculated from the pore density:

$$
d_{p}=\frac{0.0254}{P P I}
$$

The specific area $a_{P S}$ can be calculated by the following formula [25].

$$
a_{P S}=\frac{3 \pi d_{f}\left(1-e^{-((1-\varepsilon) / 0.004)}\right)}{\left(0.59 d_{p}\right)^{2}}
$$

Table 2. Metal foams properties

\section{Numerical procedure and model validation}

The numerical model is solved using a finite element analysis with COMSOL Multiphysics 5.3 (a software) [30]. 
The developed model is validated by comparing the predicted results with the experimental data from the study of Zhao et al. [31]. In the experimental study conducted by Zhao et al. [31], a copper foam sample with dimension $(200 \mathrm{~mm}, 120 \mathrm{~mm}$ and $25 \mathrm{~mm})$, pore density of 10PPI, and $95 \%$ porosity filled with paraffin wax RT58, is heated with a constant heat of $1600 \mathrm{~W} / \mathrm{m} 2$. The heat loss coefficient generated by the natural convection of air is estimated at about $3 \mathrm{~W} / \mathrm{m}^{2}$. Table 1 presents the thermophysical properties of RT58 [31]. The experimental device developed by Zhao is presented in Fig. 3. The proprieties of RT58 are given in Table 1. Fig. 4. presents a comparison between the calculated temperature evolutions of the PCM at $8 \mathrm{~mm}$ away from the bottom of the copper foam composite and experimental data. It can be observed from the figure that in the melting process, from $1200 \mathrm{~s}$ to $4000 \mathrm{~s}$, there is a small discrepancy between the results of the present model and the experimental model, as well as after the melting process. It can be concluded that the results obtained from the present model agree well with the experimental data on the whole, and the present model is reliable and reasonable.

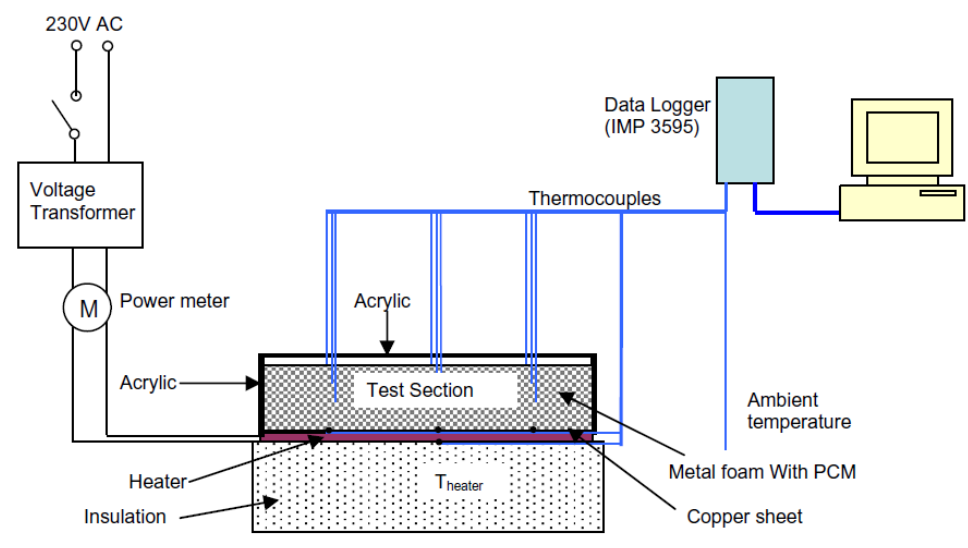

Fig. 2. Experimental device [29]

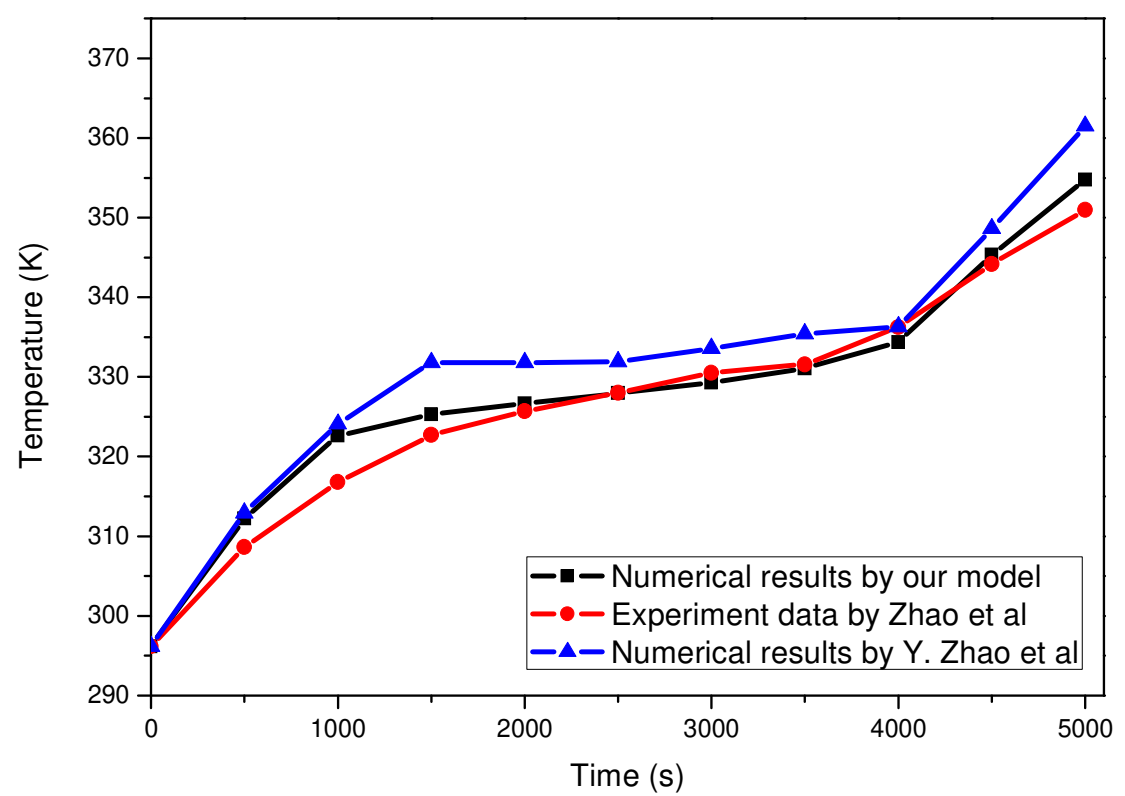

Fig. 3. Comparison between the numerical results and the literature experiment data

\section{Results and discussion}

In this study, Stephan number $S$ te, Fourrier number $F o$, Nusselt number $N u$, Rayleigh number $R a$ and a dimensionless time $\tau$ are introduced:

$$
\begin{gathered}
\text { Ste }=\frac{C_{P P}\left|T_{w}-T_{b}\right|}{L_{f}} \\
F o=\frac{\alpha_{P} t}{H^{2}}
\end{gathered}
$$




$$
\begin{aligned}
R a & =\frac{g \beta\left|T_{w}-T_{b}\right| H^{3}}{\alpha_{P} v} \\
\tau & =\text { SteFo }
\end{aligned}
$$

Nusselt number in the hot wall [12]:

$$
\left.N u\right|_{x=0}=\left.N u_{S}\right|_{x=0}+\left.N u_{P}\right|_{x=0}=-(1-\varepsilon) \frac{k_{S}}{k_{P}} \frac{\left.\int_{0}^{H} \frac{\partial T_{S}}{\partial x}\right|_{x=0} d y}{T_{w}-T_{m}}-\varepsilon \frac{\left.\int_{0}^{H} \frac{\partial T_{P}}{\partial x}\right|_{x=0} d y}{T_{w}-T_{m}}
$$

With, $\alpha$ thermal diffusivity and $v$ kinetic viscosity.

\subsection{Effects of metal foam thermal conductivity on the phase change kinetic}

To study the effect of the thermal conductivity of metal foam on the melting rate, three foams of different materials are investigated: Aluminum, copper, and nickel, with 0.93 porosity and 40PPI pore density. Fig. 4. presents the effect of the foam's matrix material on the PCM melting rate. The results show that the foam's material plays an essential role in the melting rate. The more conductive the material, the faster the melting rate. In any given moment, the liquid fraction of copper/PCM composite is more than the aluminum/PCM composite and nickel/PCM composite. After 1890 seconds, the entire PCM embedded in copper foam was liquid compared with $60 \%$ of PCM embedded in aluminum foam and 37\% of PCM embedded in nickel foam. The dimensionless time difference of melting is almost proportional to the ratio between the thermal conductivities, such as the ratio between thermal conductivity of copper and aluminum at 0.54 , and copper and nickel 0.17 .

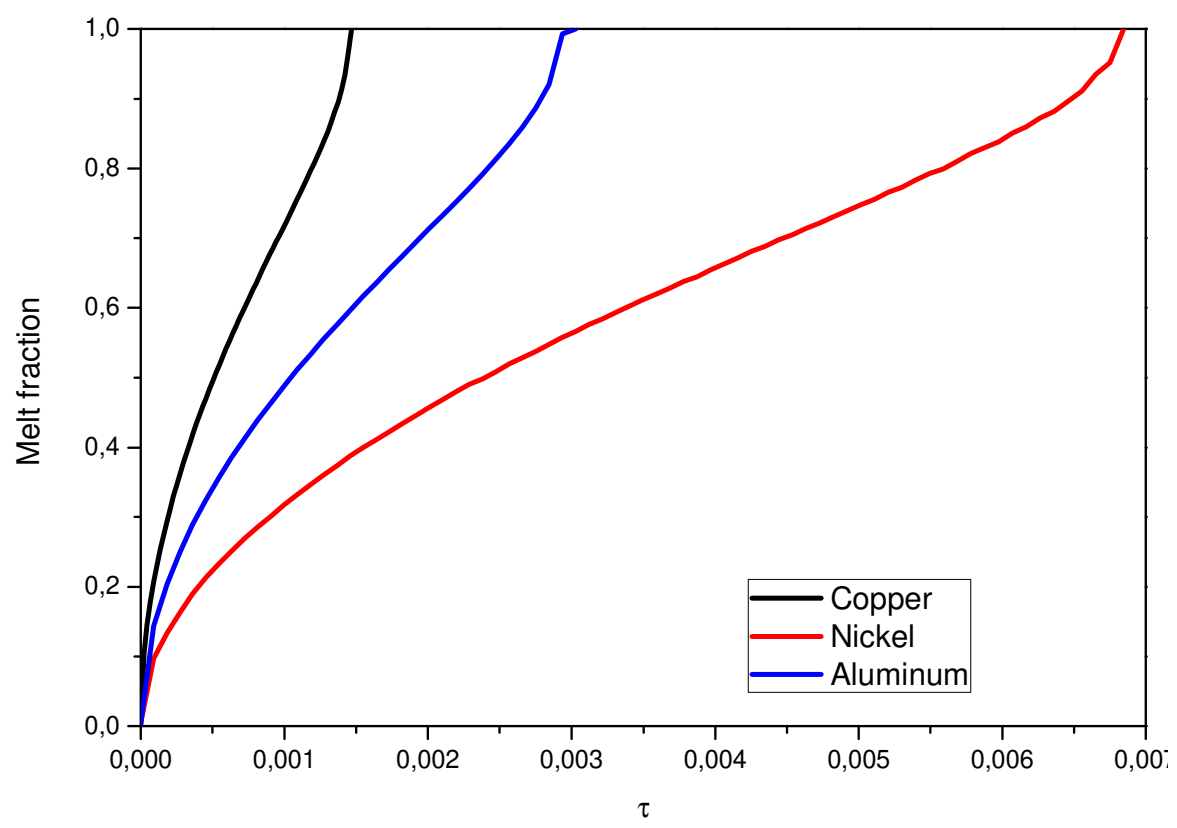

Fig. 4. Effect of metal foam on the melting rate 


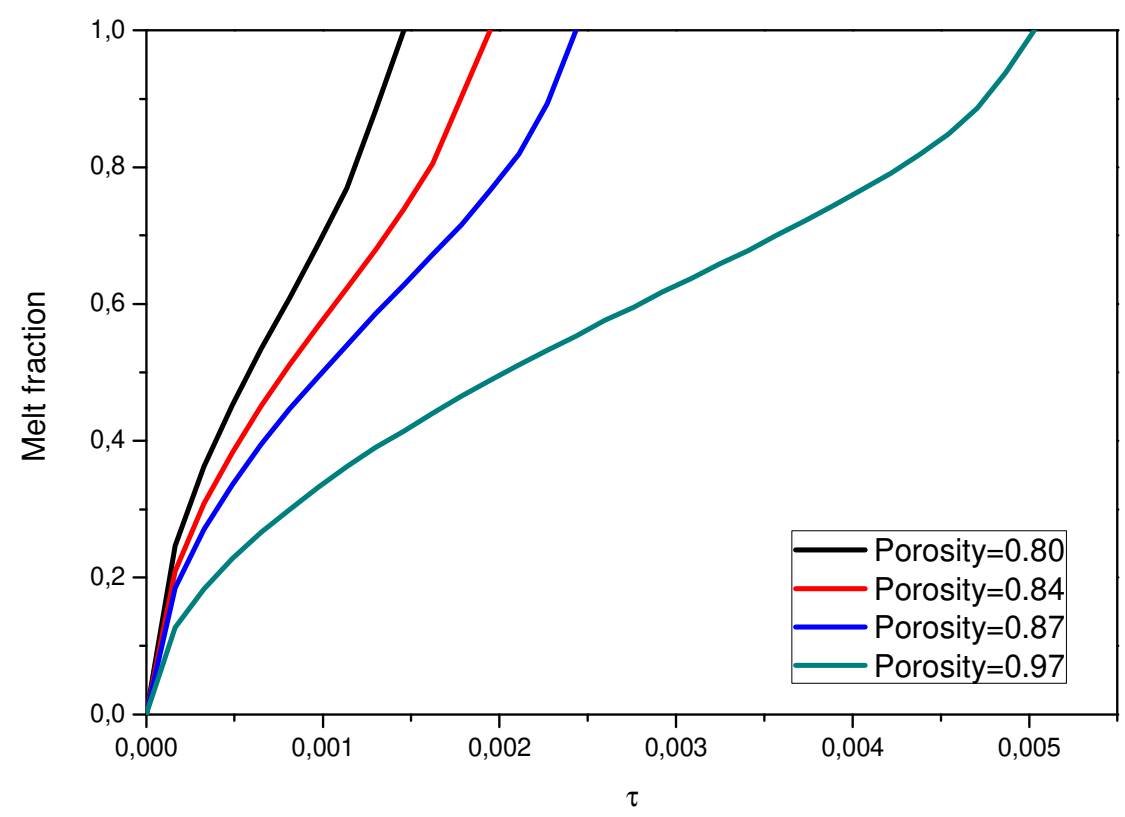

Fig. 5. The effects of porosity on the melting of PCM

\subsection{Effects of metal foam morphology}

\subsubsection{Effects of porosity on the phase change kinetic}

The aluminum foam with 40PPI is chosen to discuss the effects of porosity. Fig. 5. presents the melting rate of PCM for different porosities $(0.80,0.84,0.87$ and 0.93$)$. It is found that the PCM melts more quickly when the porosity decreases. Small porosity means more metal volume fraction with much higher thermal conductivity, which promotes better heat transfer. It was also found that the difference between the melting times is smaller when the porosity decreases. This can be explained by the fact that lower porosity leads to lower permeability (see Eq. 20), which weakens or suppresses natural convection, thus weakening the overall heat transfer, even though the conduction through the ligament plays a dominant role. Fig. 6. presents the evolution of the liquid fraction of PCM for different porosities during the solidification process. A similar process to melting was found. The PCM with small porosity solidifies more quickly, and the difference in solidification time is smaller when porosity decreases.

Fig. 7. presents the Nusselt number on the left hot wall. At first, it is larger with small porosities, but it decreases faster with larger porosities. The exchange between the sample and the hot wall with a small porosity decreases faster than with a larger porosity. This means that the natural convection of small porosities tends to be weaker.

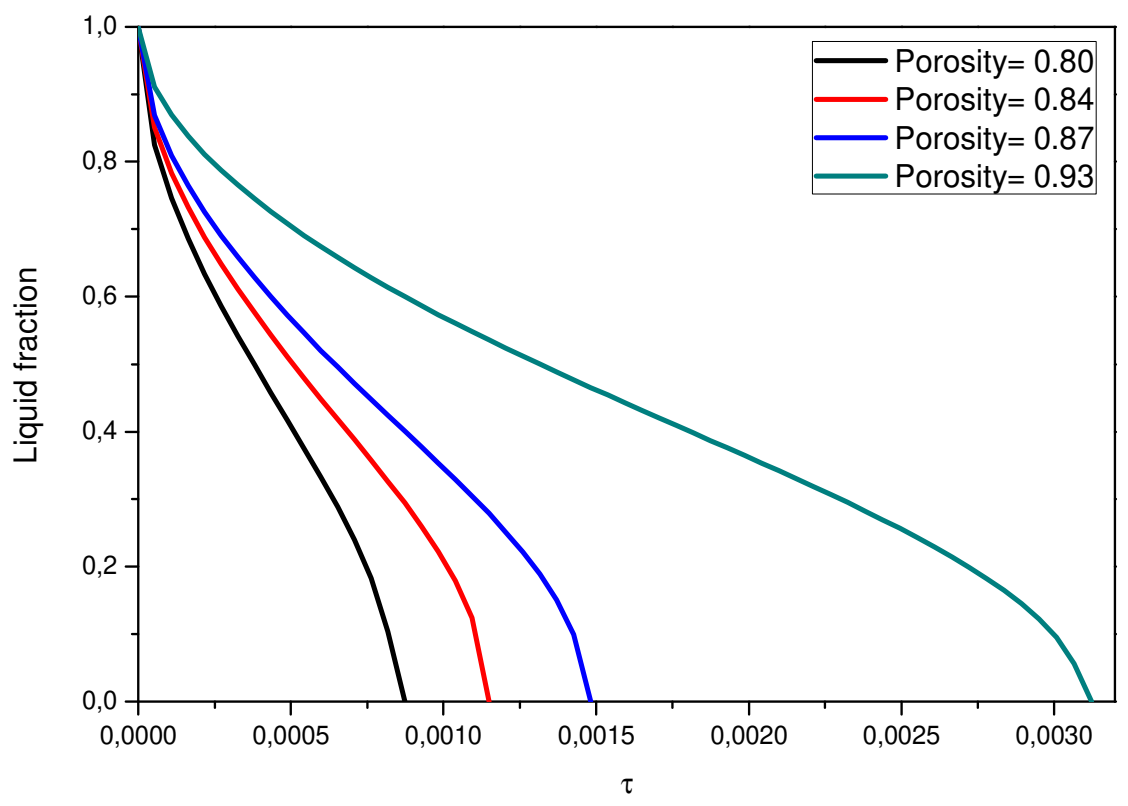

Fig. 6. The effects of porosity on the solidification of PCM 


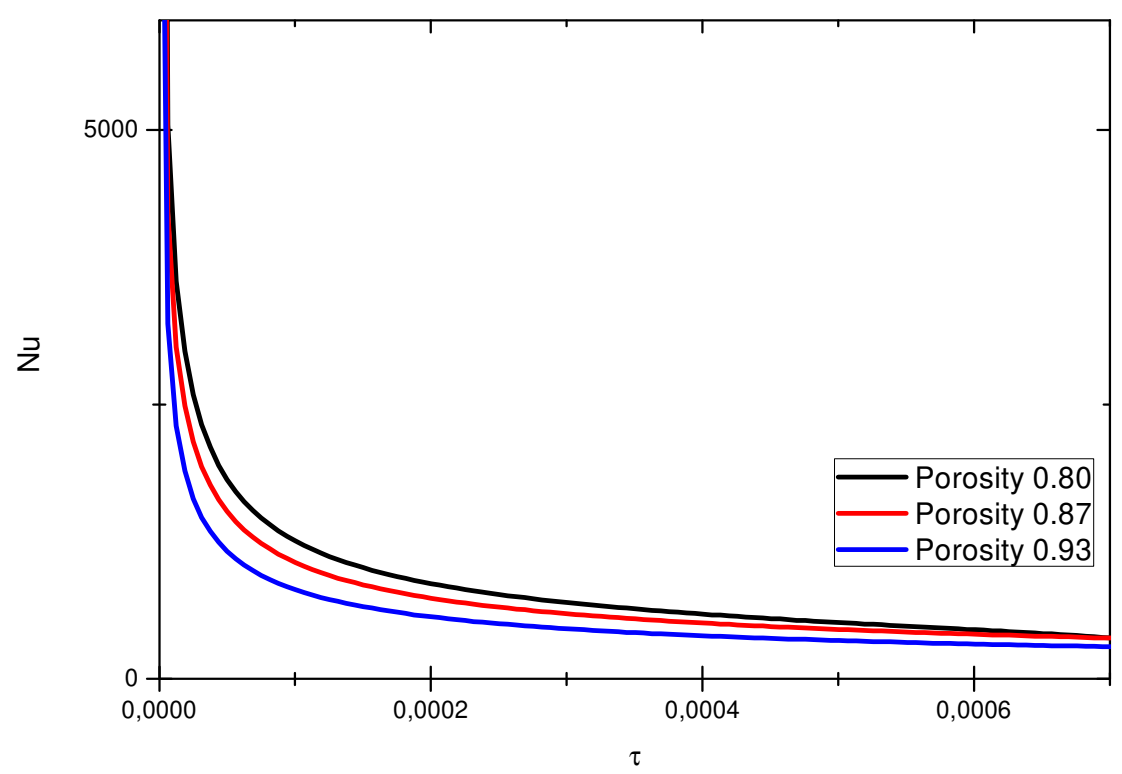

Fig. 7. The evolution of the Nusselt number on the hot wall

\subsubsection{Effects of pore density on the phase change kinetic}

In this study, four different pore densities are investigated: 5PPI, 10PPI, 20PPI and 40PPI (see Fig. 8). It was found that the PCM melts more quickly with increased pore densities. Besides, the melting time difference decreases when pore density increases. When pore density becomes big, the difference is small. High pore densities mean larger interfacial surfaces to energy exchange between PCM and foam matrix, and low permeability (Eq 20). The low value of permeability leads to suppressed natural convection. Fig. 9. presents the effect of pore density on the liquid fraction of PCM evolution during solidification. A similar process to melting was found. The PCM embedded in metal foam with high pore density solidifies more quickly, and the difference between the solidification time is smaller when pore density increases.

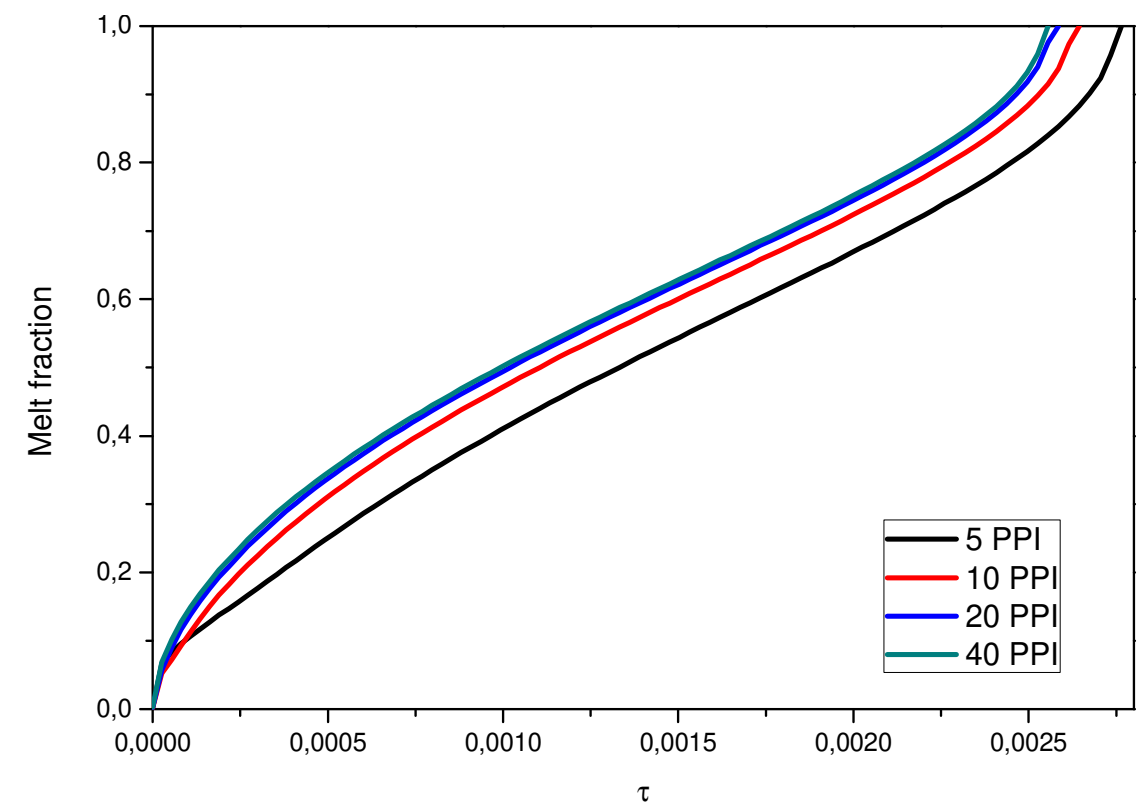

Fig. 8. The effect of pore density on the melting of PCM 


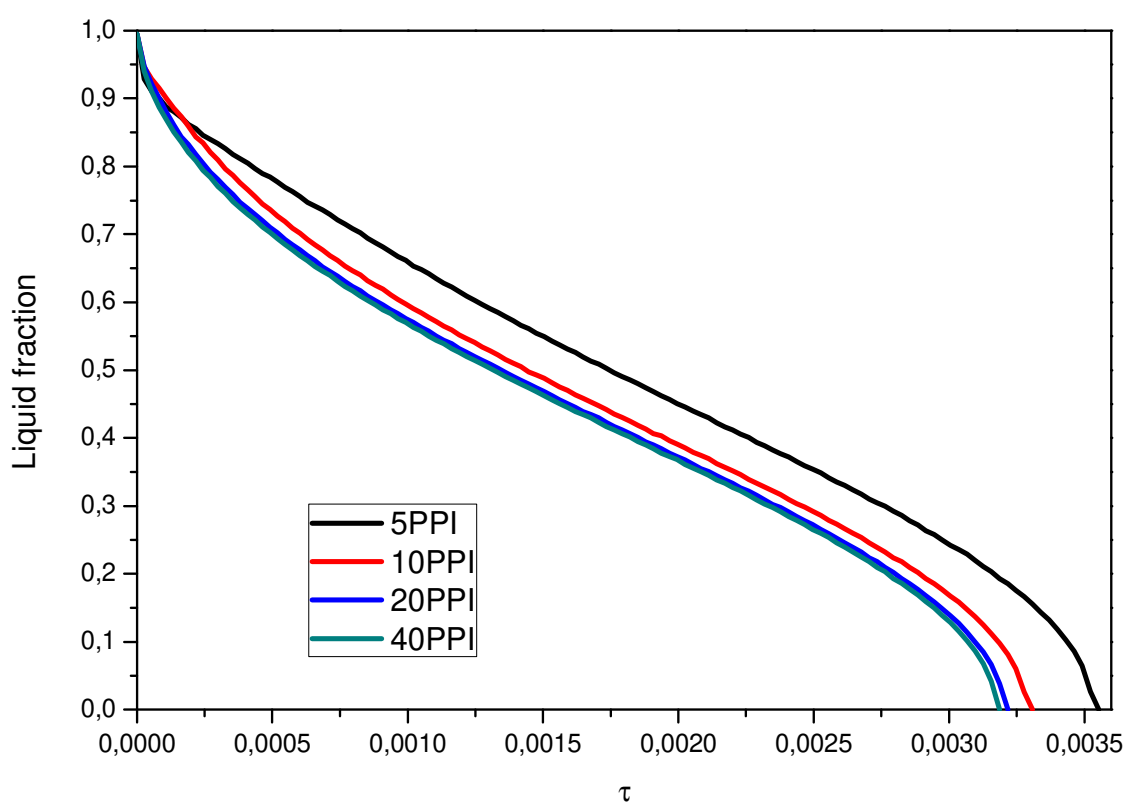

Fig. 9. The effect of pore density on the solidification of PCM

\subsection{Effects of heating and cooling conditions on the phase change kinetic}

To study the effect of heating and cooling conditions on the kinetic of phase change of PCM filled in metal foam, a cyclic heating and cooling study was carried out. In this section, a study on the cyclic heating and cooling of $\mathrm{PCM} / \mathrm{metal}$ foam composite used as an energy storage system is presented. There are two types of cyclic heating and cooling applied to the paraffin RT27/aluminum foam $(0.93,40 \mathrm{PPI})$. The first one is a constant flux of $+1800 \mathrm{~W} / \mathrm{m}^{2}$ provided at the heated wall for $60 \mathrm{~min}$, and then its value becomes negative at the same magnitude for the next $60 \mathrm{~min}$. The second is a sine function having the similar area under the curve, and provided for the same time, as shown in Fig. 10. The equations of the boundary condition are presented in Eq. (30).

$$
\begin{aligned}
& q_{w}=\left\{\begin{array}{l}
1800 \mathrm{~W} / \mathrm{m}^{2},(0 \leq t \leq 60 \mathrm{~min}) \\
-1800 \mathrm{~W} / \mathrm{m}^{2},(60<t \leq 120 \mathrm{~min})
\end{array}, \quad\right. \text { Constant heating, cooling } \\
& q_{w}=900 \times \pi \times \sin (\pi \times t / 60),
\end{aligned}
$$

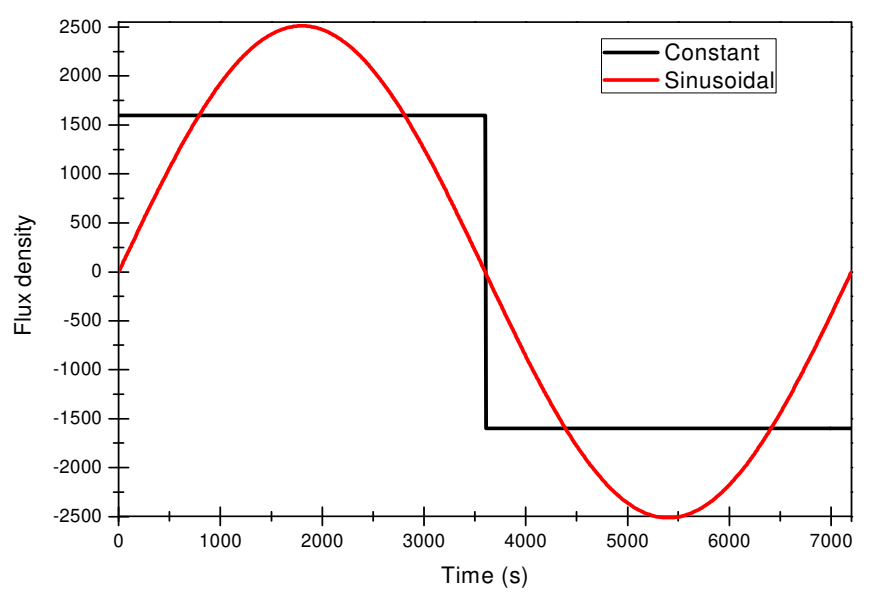

Fig. 10. Heating, cooling cycle 
Fig. 11 presents the melt fraction evolution of PCM with constant heat flux and a sinusoidal heat flux. The melting of paraffin with a constant heat flux starts after 40 seconds and with variable sinusoidal heat flux, starts after about 5 min. The melt fraction of both becomes the same after 28 minutes, and after that, the fraction with variable heat becomes higher, as shown in Fig. 5. The all paraffin filled in aluminum foam becomes liquid (Mel fraction equals 1) after heating with a sinusoidal heat flux for $41 \mathrm{~min}$, before heating with a constant heat flux, which becomes completely liquid after $48 \mathrm{~min}$. The behavior of the melt fraction evolution is due to the constant heat flux, as the heating rate is constant with magnitude $1800 \mathrm{~W} / \mathrm{m}^{2}$. Whereas in case of heating with sinusoidal heat flux, the heating ratio is zero at the start of melting and increases with time in a sinusoidal manner and becomes maximum at 30 min Eq (30). It can be seen that under the same conditions and with the same amount of energy supplied, all paraffin becomes liquid with sinusoidal heating, faster than with constant heating. In the solidification process of PCM, the solidification of PCM with sinusoidal heating starts after 10 minutes, compared to the solidification with constant heating which starts after 33 minutes. The liquid fraction is the same with constant heating and sinusoidal heating, either in the case of constant heat flux or sinusoidal heat flux, the paraffin is not completely solidified.

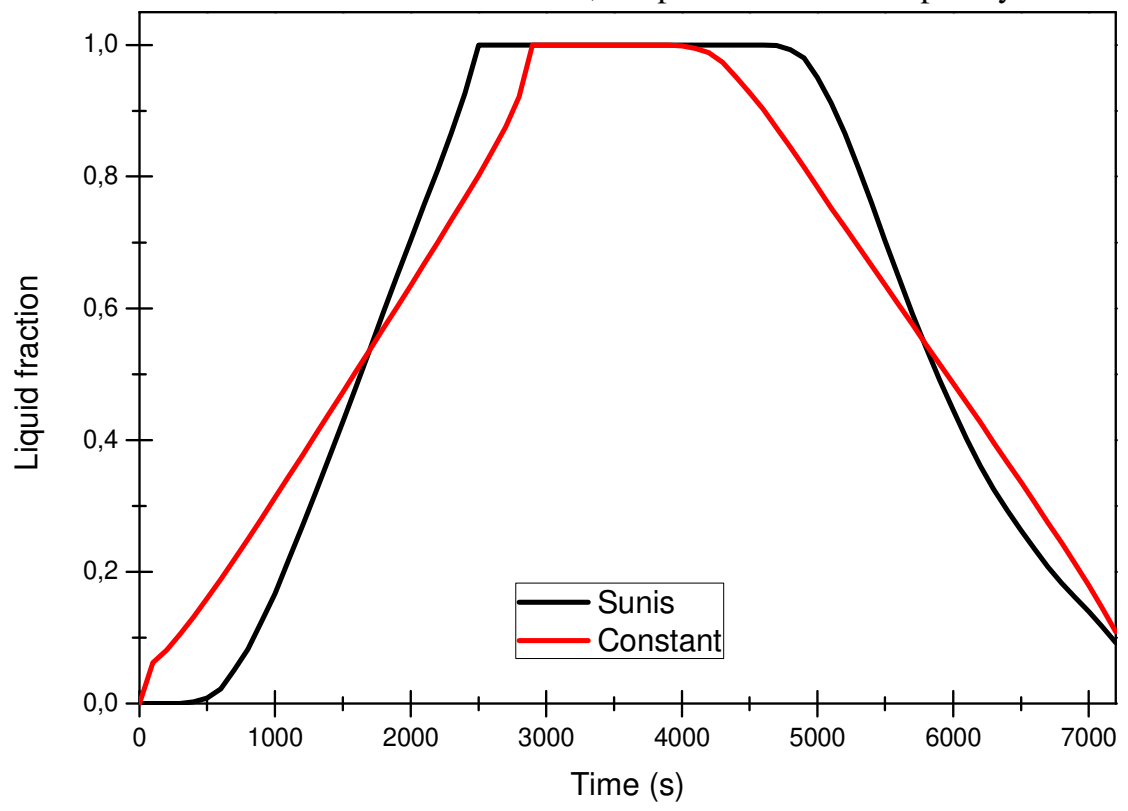

Fig. 11. Liquid fraction during melting and solidification.

\section{Conclusion}

A numerical study is carried out on the solid-liquid and liquid-solid phase change of PCM embedded in metal foam. The Brinkman-Forchheimer-extended Darcy equation and the local thermal non-equilibrium model assumption are used due to the larger difference in thermal properties between the solid matrix (metal foam) and PCM by applying a two-energy equation. The phase change is modeled by utilizing the enthalpy-porosity method. The PCM used is paraffin RT27. The numerical model was developed and validated through comparison with the literature and experimental data. The metal foam properties, thermal conductivity, porosity, and pore density effects on the kinetic of the phase change, were studied. The effect of heating/ cooling conditions were also investigated. The results show that the thermal conductivity plays an essential role in the kinetic phase change of PCM embedded in metal foam. The melting of PCM embedded in metal foam is almost proportional to the metal foam's thermal conductivity. The melt fraction of PCM embedded in metal foam increases with the decrease in porosity. The PCM melting and solidification rates for both increase with higher pore density. Small pore density increases permeability and natural convection, and large pore density leads to low permeability that weakens or suppresses natural convection, thus weakening the overall heat transfer. It is observed with sinusoidal heating that the time taken for the melting of PCM embedded in metal foam reduces compared to constant heating. In the solidification process of PCM, the solidification of PCM with sinusoidal heating starts later, compared to solidification through constant heating. However, the liquid fraction is almost the same as a result and the PCM is not completely solidified in either case. This study is a good motivation for more quantitative studies on efficiency and time optimization of the storage and release process.

\section{References:}

[1]: Du K, Calautit J, Wang Z, Wu Y, Liu H. A review of the applications of phase change materials in cooling, heating and power generation in different temperature ranges. Applied Energy 220 (2018) 242-273. 
[2]: Lisa Boussaba, Amina Foufa, Said Makhlouf, Gilles Lefebvre, Laurent Royon, Elaboration and properties of a composite bio-based PCM for an application in building envelopes, Construction and Building Materials 185 (2018) $156-165$.

[3]: Zalba B, Marın JM, Cabeza LF, Mehling H, Review on thermal energy storage with phase change: materials, heat transfer analysis and applications, Applied Thermal Engineering 23 (2003) 251-283.

[4]: Chenzhen J, Zhen Q, Zhenghua L, Swapnil D, Fook HC, Fei Duan, Non-uniform heat transfer suppression to enhance PCM melting by angled fins, Applied Thermal Engineering 129 (2018) 269-279.

[5]: Jegadheeswaran S, Pohekar SD, Kousksou T, Conductivity particles dispersed organic and inorganic phase change materials for solar energy storage-an exergy based comparative evaluation, Energy Procedia 14 (2012) 643648.

[6]: Siahpush A, O’Brien J, Crepeau J, Phase change heat transfer enhancement using copper porous foam, Journal of Heat Transfer 130 (2008) 082301-1-11.

[7]: Xiao X, Zhang P, Li M, Preparation and thermal characterization of paraffin/metal foam composite phase change material, Applied Energy 112 (2013) 1357-1366.

[8]: Rehman T, Ali HM, Janjua MM, Sajjad U, Yan WM, A critical review on heat transfer augmentation of phase change materials embedded with porous materials/foams, International Journal of Heat and Mass Transfer 135 (2019) 649-673.

[9]: Xiao X, Zhang P, Li M, Effective thermal conductivity of open-cell metal foams impregnated with pure paraffin for latent heat storage, International Journal of Thermal Sciences 81 (2014) 94-105.

[10]: Huang X, Lin Y, Alva G, Fang G, Thermal properties and thermal conductivity enhancement of composite phase change materials using myristyl alcohol/metal foam for solar thermal storage, Solar Energy Materials and Solar Cells 170 (2017) 68-76.

[11]: Sundarram SS, Li W, The effect of pore size and porosity on thermal management performance of phase change material infiltrated microcellular metal foams, Applied Thermal Engineering 64 (2014) 147-154.

[12]: Zhao Y, Zhao CY, Xu ZG, Xu HJ, Modeling metal foam enhanced phase change heat transfer in thermal energy storage by using phase field method, International Journal of Heat and Mass Transfer 99 (2016) 170-181.

[13]: Abishek S, King AJ, Nadim N, Mullins BJ, Effect of microstructure on melting in metal-foam/paraffin composite phase change materials, International Journal of Heat and Mass Transfer 127 (2018) 135-144.

[14]: Aadmi M, Karkri M, El Hammouti M, Heat transfer characteristics of thermal energy storage of a composite phase change materials: numerical and experimental investigations, Energy 72 (2014) 381-392.

[15]: Whitaker S, The method of volume averaging. Vol. 13. Dordrecht: Springer Netherlands; 1999.

[16]: Annabelle Joulin, Zohir Younsic, Laurent Zalewski, Stéphane Lassue, Daniel R. Roussed,Jean-Paul Cavrot, Experimental and numerical investigation of a phase change material: thermal-energy storage and release, Applied Energy 88 (2011) 2454-2462.

[17]: Viswanath R, Jaluria Y, A comparison of different solution methodologies for melting and solidification problems in enclosures, Numerical Heat Transfer 24. Part B. (1993) 77-105.

[18]: Yao Y, Wu H, Liu Z, Gao Z, Pore-scale visualization and measurement of paraffin melting in high porosity open-cell copper foam, International Journal of Thermal Sciences 123 (2018) 73-85.

[19]: Biwole PH, Groulx D, Souayfane F, Chiu T, Influence of fin size and distribution on solid-liquid phase change in a rectangular enclosure, Applied Thermal Engineering 124 (2018) 433-446.

[20]: Kheirabadi AC, Groulx D, Simulating phase change heat transfer using COMSOL and fluent: effect of the mush-zone constant, Computational Thermal Sciences 7 (2015) 427-440.

[21]: Zukauskas AA, Convective heat transfer in cross-flow, In: Kakac S, Shah RK, Aung W, editors, Handbook of single-phase convective heat transfer. New York: Wiley; 1987.

[22]: Boomsma K, Poulikakos D, On the effective thermal conductivity of a three dimensionally structured fluidsaturated metal foam, International Journal of Heat and Mass Transfer 44 (2001) 827-836.

[23]: Du Plessis P, Montillet A, Comiti J, Legrand J, Pressure drop prediction for flow through high porosity metallic foams, Chemical Engineering Science 49 (1994) 3545-3553.

[24]: Calmidi VV, Transportation phenomena in high porosity fibrous metal foams [PhD thesis]. University of Colorado; 1998.

[25]: Bhattacharya A, Calmidi VV, Mahajan RL, Thermophysical properties of high porosity metal foams, International Journal of Heat and Mass Transfer 45 (2002) 1017-1031.

[26]: Tadrist L, Miscevic M, Rahli O, Topin F, About the use of fibrous materials in compact heat exchangers, Experimental Thermal and Fluid Science 28 (2004) 193-199.

[27]: Dukhan N, Correlations for the pressure drop for flow through metal foam, Experiments in Fluids 41 (2006) 665-672. 
[28]: Yang X, Bai J, Lu T, A simplistic analytical model of permeability for open-cell metallic foams, Chinese Journal of Theoretical and Applied Mechanics 46(6) (2014) 982-986.

[29]: Calmidi VV, Mahajan EL, Forced convection in high porosity metal foams, Journal of Heat Transfer 122(3) (2000) 557-565.

[30]: COMSOL Multiphysics user's guide. COMSOL 2015. Available from: URL: http://www.comsol.com.

[31]: Zhao CY, Lu W, Tian Y, Heat transfer enhancement for thermal energy storage using metal foams embedded within phase change materials (PCMs), Solar Energy 84 (2010) 1402-1412. 